\title{
Crude oil biodegradation using isolated bacteria from oil polluted soil
}

\begin{abstract}
Contamination due to the spillage of petroleum or petroleum-derived hydrocarbons on different ecosystems has become critical issues to environment and living things. The use of native bacteria as an environmental friendly treatment of hydrocarbons pollution is very promising. The objective of the study is to identify the native hydrocarbon-degraders isolated from oil refinery. Bacteria from crude oil were isolated and cultured in enrichment media. The isolated strains were then cultured in different oil concentration. The isolated bacterial strains were Gram-stained and further identified via 16S rRNA sequencing. The resultant sequences were then aligned with the available online database for identification of the bacteria. The isolated bacteria belonged to families of Pseudomonadaceae and Moraxellaceae (Acinetobacter genus). The bacteria show high degradation of hydrocarbon and can be used to remediate polluted soils in tropical environment.
\end{abstract}

Keyword: Oil pollution; Bacterial bioremediation; Hydrocarbon polluted soils 